\title{
Improved Survival beyond 28 Days up to 1 Year after CytoSorb Treatment for Refractory Septic Shock: A Propensity-Weighted Retrospective Survival Analysis
}

\author{
Willem P. Brouwer ${ }^{a, b}$ Servet Duran ${ }^{c}$ Can Ince $^{d}$ \\ aDepartment of Gastroenterology \& Hepatology, Erasmus MC, University Medical Center Rotterdam, Rotterdam, \\ The Netherlands; bepartment of Internal Medicine, Maasstad Ziekenhuis, Rotterdam, The Netherlands; \\ 'Department of Intensive Care Medicine, Maasstad Ziekenhuis, Rotterdam, The Netherlands; ${ }^{\mathrm{d} D e p a r t m e n t}$ of \\ Intensive Care Medicine, Erasmus MC, University Medical Center Rotterdam, Rotterdam, The Netherlands
}

\section{Keywords}

Sepsis · Septic shock · Treatment · Hemofiltration · CytoSorb · Cytosorbent $\cdot$ Mortality $\cdot$ Outcome

\begin{abstract}
Background and Aims: It is currently unknown whether CytoSorb treatment for septic shock improves long-term survival beyond 28 days from intensive care unit (ICU) admission and which factors determine outcome. Methods: This was a long-term follow-up retrospective analysis of patients with septic shock who were treated with continuous renal replacement therapy (CRRT) + CytoSorb $(n=67)$ or CRRT alone $(n=49)$. These patients were previously analyzed for 28-day mortality. The primary outcome was the time to longterm all-cause mortality. Factors associated with time to event were analyzed both weighted by stabilized inverse probability of treatment weights (sIPTW) as well as unweighted stratified by therapy received. Results: The median follow-up for the total cohort was 30 days (interquartile range [IQR]: 5-334, maximum 1,059 days) after ICU admission and 333 days (IQR: 170-583) for those who survived beyond 28 days ( $n=59$ ). Survival beyond 28 days was sustained up to 1 year after ICU admission for both treatment regimens: $80 \%$ (standard error [SE] 7\%) vs. $87 \%$ (SE 7\%), for CytoSorb vs. CRRT, respectively, $p=0.853$. By sIPTW, CytoSorb
\end{abstract}

was significantly associated with long-term outcome compared to CRRT (adjusted hazard ratio [aHR] 0.59, 95\% confidence interval [Cl] 0.37-0.93, $p=0.025$ ). Independent factors associated with long-term outcome in CytoSorb-treated patients were baseline $\log _{10}$ lactate levels (aHR 5.1, $p=0.002$ ), age in the presence of comorbidity (aHR 2.60, $p=0.013$ ), and presence of abdominal sepsis (aHR 0.34, $p=0.004$ ). A lactate level above $6.0 \mathrm{mmol} / \mathrm{L}$ at the start of CytoSorb therapy had a positive predictive value of $79 \%$ for mortality $(p=0.013)$. Conclusions: Survival is achieved with CytoSorb and CRRT for patients with septic shock beyond 28 days from ICU admission and may be improved for CytoSorb treatment. Lactate levels above $6.0 \mathrm{mmol} / \mathrm{L}$ at the start of CytoSorb therapy are predictive of worse outcome with high specificity and positive predictive value.

(c) 2020 The Author(s).

Published by S. Karger AG, Basel

\section{Introduction}

Worldwide, approximately 45 million patients suffer from sepsis with an estimated 11 million deaths each year [1-4]. Sepsis and septic shock are the leading causes of mortality with an amplified incidence due to increased aging of populations, comorbidities, and greater awareness of sepsis, but also because of emerging pandemics [5, 
6]. Septic shock arises from a derailed host response to infection, with consequently a condition what is known as a "cytokine storm" leading to shock [6]. Sepsis is generally treated with early resuscitation and antibiotics, which is strongly associated with an improved outcome [5]. More innovative treatment options have been evaluated, most with disappointing results. Continuous renal replacement therapy (CRRT) proved able to clear cytokines from serum and possibly improve the cytokine storm; however, a clinical benefit seems to be lacking [7-9]. Recently, we have shown that CytoSorb treatment may lead to an improved clinical outcome at 28 days from intensive care unit (ICU) admission for refractory septic shock [10]. CytoSorb has been developed and approved for use in patients with severe cytokinemia [11-13]. It is an adsorber which can be used in addition to CRRT and has been shown to be able to reduce circulating cytokines by $>90 \%$ [13]. It is currently not known whether CytoSorb therapy for refractory septic shock is associated with an improved survival beyond the first month of ICU admission. Moreover, factors determining (un)favourable outcomes and the optimal timing of CytoSorb therapy are currently not known. Therefore, the aim of the current study was to investigate the long-term outcome of the septic shock patients we had previously investigated for 28-day survival who were treated with CytoSorb and/or CRRT and to study factors associated with outcome in CytoSorb-treated patients.

\section{Patients and Methods}

\section{Patients}

This is a long-term follow-up of a retrospective cohort study, in which patients were treated at the ICU for septic shock with CytoSorb and/or CRRT. Here, we further studied the cohort of patients we had previously studied for 28-day survival. The selection methods and inclusion and exclusion criteria are described elsewhere in detail [10]. In short, patients admitted to the ICU with septic shock who were treated with CytoSorb or CRRT in the period from January 1, 2014, to April 1, 2017, were eligible for inclusion in the study. These patients were treated at the discretion of the attending intensive care physician according to local protocol. Patients were excluded from the analysis in case the primary reason for therapy was not septic shock. In case of improvement of septic shock or renal function, CytoSorb or CRRT was discontinued. CytoSorb was used according to the manufacturer's protocol. It was placed in a blood-pump circuit with an optimal ultrafiltration rate of $250-400 \mathrm{~mL} / \mathrm{min}$. The CytoSorb adsorber was changed after 24 hours of use.

\section{Definitions}

Septic shock was defined as persisting hypotension requiring vasopressive medication to maintain mean arterial pressure
(MAP) $\geq 65 \mathrm{mmHg}$ and having a serum lactate level of $>2 \mathrm{mmol} / \mathrm{L}$ despite adequate volume resuscitation [6]. Shock reversal was defined as a serum lactate level of $\leq 2 \mathrm{mmol} / \mathrm{L}$ and discontinuation of vasopressive medication [14].

\section{Endpoints}

The primary endpoint was the time to all-cause mortality compared for CytoSorb versus CRRT alone. Also, factors associated with all-cause mortality during long-term follow-up were analyzed separately. All-cause mortality was measured from ICU admission until the last follow-up date known (irrespective of ICU, in-hospital, or out-of-hospital mortality).

\section{Statistical Analysis}

A survival analysis was employed with application of the inverse probability of treatment weights (IPTW), including a stabilizing method to avoid bias from extreme weights. Stabilized IPTW (sIPTW) is applied to overcome differences in baseline patient characteristics, to mimic a randomized controlled trial [15]. The sIPTW was previously constructed [10] and contains the following variables at the start of therapy: age, presence of any comorbidity, SOFA score, lactate level, noradrenalin level, type of sepsis, and medical or surgical treatment. Since mean arterial pressure (MAP) at the start of therapy was at imbalance between the 2 treatment groups and associated with outcome, it was additionally accounted for in the final analysis $[10,15]$. Factors associated with all-cause mortality in CytoSorb-treated patients were analyzed as well without application of sIPTW. For this, factors with a $p$ value of $<0.2$ in univariate Cox regression analysis were considered for multivariate analyses, where a full, forced and final model was constructed. Using the same variables from the full model, the final model was constructed using the back step and forward step likelihood ratio method, where the model with the highest $\chi^{2}$ value was selected. Moreover, factors determining outcome were selected and inspected for optimal clinical use by analyzing tests' characteristics with Harrell's C-statistic and for laboratory values choosing the optimal cutoff using the highest sensitivity and specificity sum. Laboratory values and variables with a skewed normal distribution were log-transformed prior to the analysis. SAS Enterprise version 9.4 and SPSS version 22.0 (SPSS Inc., Chicago, IL, USA) were used to perform the statistical analyses. All statistical tests were twosided and evaluated at the 0.05 level of significance.

\section{Results}

\section{Patient and Follow-Up Characteristics}

In total, 67 patients with septic shock were treated with CytoSorb, versus 49 with CRRT. Table 1 shows the patient characteristics at the start of therapy [10]. Patients were followed for a median duration of 30 days (interquartile range [IQR]: 5-334) with a maximum of 1,059 days after ICU admission. For those patients followed beyond 28 days after ICU admission $(n=59)$, the median follow-up duration was 333 days (IQR: 170-583). There was no difference in median follow-up duration between the 2 treatment groups $(p=0.880)$. Median ICU and hospital admission were 9 and 
Table 1. Patient characteristics at the start of therapy*

\begin{tabular}{|c|c|c|c|}
\hline Characteristics & $\begin{array}{l}\text { CytoSorb } \\
(n=67)\end{array}$ & $\begin{array}{l}\text { CRRT only } \\
(n=49)\end{array}$ & $\begin{array}{l}\text { sIPTW-adjusted } \\
p \text { value }\end{array}$ \\
\hline \multicolumn{4}{|l|}{ Demography } \\
\hline Age, years, mean (SD) & $61.1(14.7)$ & $68.7(9.6)$ & 0.410 \\
\hline Male, $n(\%)$ & $37(55)$ & $30(61)$ & 0.008 \\
\hline Any comorbidity, $n(\%)$ & $43(64)$ & $42(88)$ & 0.601 \\
\hline Diabetes mellitus type 2 & $14(21)$ & $20(41)$ & 0.778 \\
\hline Hypertension & $23(34)$ & $26(53)$ & 0.409 \\
\hline Coronary heart disease & $9(13)$ & $8(16)$ & 0.064 \\
\hline Heart failure (systolic/diastolic) & $4(6)$ & $10(20)$ & 0.501 \\
\hline Prior chronic kidney disease & $8(12)$ & $18(37)$ & 0.002 \\
\hline Peripheral artery disease & $10(15)$ & $8(16)$ & 0.021 \\
\hline Cerebrovascular accident & $6(9)$ & $3(6)$ & 0.369 \\
\hline COPD & $8(12)$ & $10(20)$ & 0.002 \\
\hline Surgical (otherwise medical), $n(\%)$ & $27(40)$ & $6(12)$ & 0.930 \\
\hline \multicolumn{4}{|l|}{ Sepsis origin, $n(\%)$} \\
\hline Abdominal sepsis & $31(46)$ & $12(25)$ & 0.809 \\
\hline Pneumosepsis & $14(21)$ & $21(43)$ & 0.914 \\
\hline Others & $22(33)$ & $16(33)$ & 0.883 \\
\hline \multicolumn{4}{|l|}{ Hemodynamics, mean (SD) } \\
\hline Lactate level, mmol/L & $6.9(5.6)$ & $2.9(3.1)$ & 0.560 \\
\hline Noradrenaline, $\mu \mathrm{g} / \mathrm{kg} / \mathrm{min}$ & $0.96(0.73)$ & $0.28(0.36)$ & 0.943 \\
\hline Mean arterial pressure & $69(15)$ & $77(18)$ & 0.015 \\
\hline SOFA at start of treatment & $13.8(2.8)$ & $12.8(3.2)$ & 0.164 \\
\hline
\end{tabular}

CRRT, continuous renal replacement therapy; sIPTW, stabilized inverse probability of treatment weights. * Table 1 characteristics have been previously published elsewhere [10].

17-22 days, respectively, for both groups ( $p=0.735$ and $p=0.991)$. The mean duration from ICU admission to the start of treatment was 2.1 (SE 0.36) versus 1.66 (SE 0.38) days ( $p=0.416)$ for CRRT versus CytoSorb, respectively. In the CRRT group, CRRT was given for a mean duration of 4.96 (SE 0.63) days versus 4.97 (SE 0.55) days in the CytoSorb group $(p=0.990)$. The mean treatment duration for the CytoSorb group was 2.34 (SE 0.16) days.

\section{CytoSorb and CRRT Lead to a Durable Response}

beyond 28 Days of ICU Admission

For the total group, at 1 year after ICU admission, the unweighted survival rate was similar in both groups with CytoSorb-treated patients being 42\% (standard error [SE] 6\%) and CRRT-treated patients, 43\% (SE 7\%) (Fig. 1a), while CytoSorb-treated patients had a more severe shock as mentioned before. For those patients who survived beyond 28 days of ICU admission, survival for CytoSorb and CRRT at 1 year after ICU admission was comparable at $80 \%$ (SE 7\%) and $87 \%$ (SE 7\%), respectively $(p=0.853)$.

CytoSorb Improves Long-Term Survival after Septic Shock

\section{CytoSorb May Improve Long-Term Outcome} Compared to CRRT Alone

By Kaplan-Meier analysis applying sIPTW, the 1-year survival was 37\% (SE 7\%) versus 26\% (SE 12\%) for CytoSorb versus CRRT, respectively (log-rank $p=0.21$ ). By Cox regression analysis adjusted for the MAP at baseline of therapy, CytoSorb treatment showed a significantly higher survival rate compared to CRRT (adjusted HR [aHR] 0.59, 95\% Wald confidence interval [CI]: 0.37-0.93, $p=0.025$; Fig. 1b). In this model, presence of chronic kidney disease (aHR 1.10, $p=0.736$ ) or gender (aHR 1.02, $p=0.940$ ) were not significantly associated with outcome.

\section{Factors Associated with Long-Term Outcome in the CytoSorb Group}

Factors in univariable and multivariable analyses associated with outcome in the CytoSorb group can be found in Table 2. Noradrenaline levels did not significantly interfere with lactate levels $(p=0.496)$. However, there was evidence of collinearity for noradrenaline and lactate levels. When lactate was replaced by noradrenaline, the multivariate Cox 
Fig. 1. Survival plots for CytoSorb versus CRRT therapy without $\operatorname{sIPTW}(\mathbf{a})$ and with sIPTW (b). CRRT, continuous renal replacement therapy; sIPTW, stabilized inverse probability of treatment weights; aHR, adjusted hazard ratio.

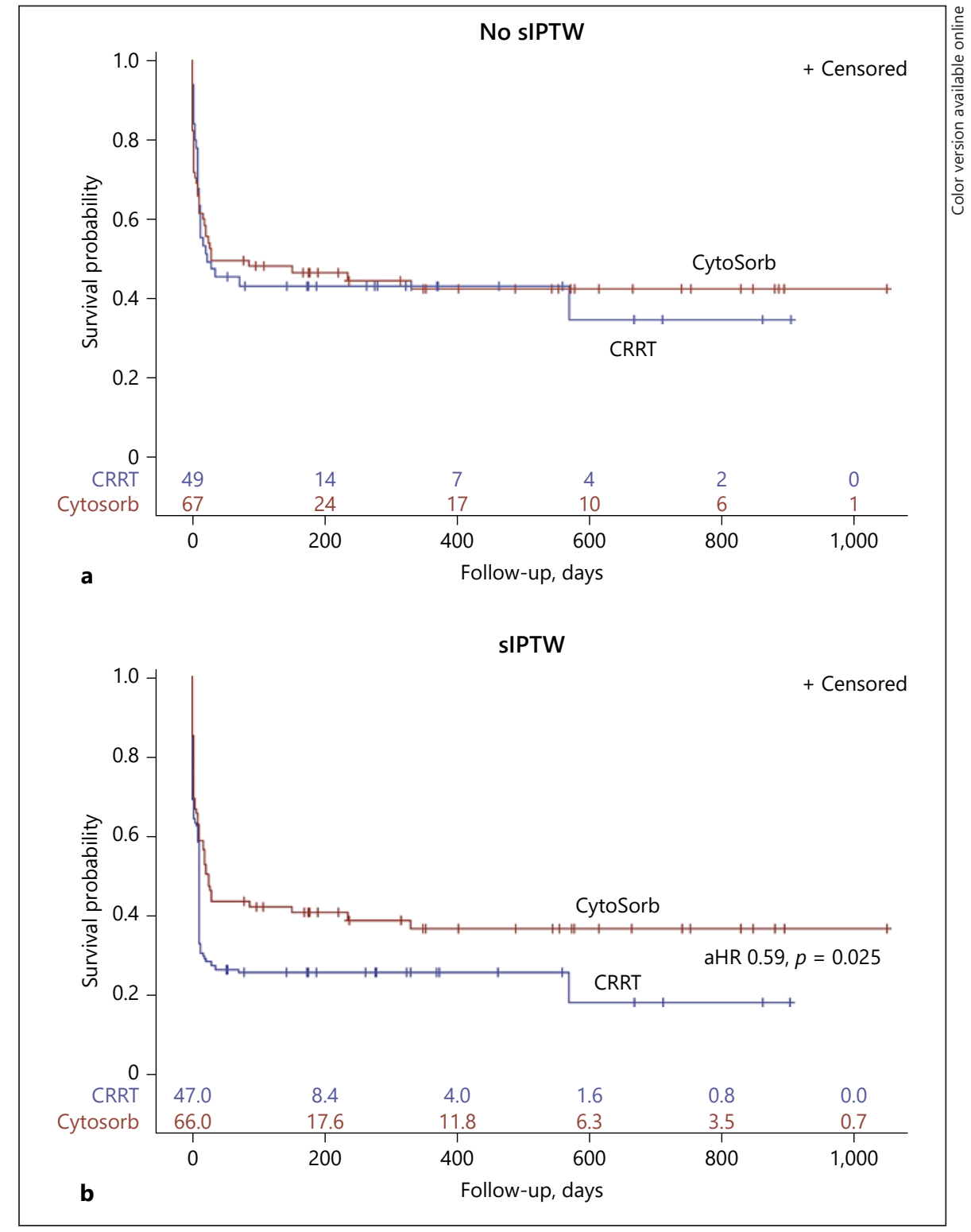

regression model showed a slight weaker $\chi^{2}$ and noradrenaline was not significantly associated with outcome (HR 2.86, 95\% CI: 0.99-8.21, $p=0.051$ ). The final model (Table 2) had a Harrell C-statistic of 0.80 (SE 0.04, $p<0.001$ ) for mortality prediction in the long term, but also in the short term (28-day mortality C-statistic 0.81 , SE $0.04, p<$ 0.001 ). For a simple and practical approach, the most important factor from this model was selected for mortality prediction: it was observed that a lactate level cutoff of 6.0 $\mathrm{mmol} / \mathrm{L}$ (HR 3.1, SE 0.33, $p<0.001$ ) had the highest specificity $(79 \%)$ and sensitivity (58\%) sum and positive predictive value (79\%) for long-term mortality, Harrell C-statistic 0.67 (95\% CI: 0.54-0.80, $p=0.013$ ). Figure 2 shows the Ka-
plan-Meier curve for the CytoSorb cohort stratified by lactate below or above $6.0 \mathrm{mmol} / \mathrm{L}$. This same cutoff of lactate level also had the highest sensitivity (63\%) and specificity (77\%) for 28 -day mortality where the positive predictive value was slightly lower (71\%).

\section{Discussion}

In this long-term follow-up study, we have shown that long-term survival with either CytoSorb or CRRT beyond 28 days of ICU admission is achievable. In a weighted analysis, CytoSorb showed an improved long-term outcome 
Fig. 2. Survival plot within CytoSorb-treated patients according to lactate level at the start of treatment.

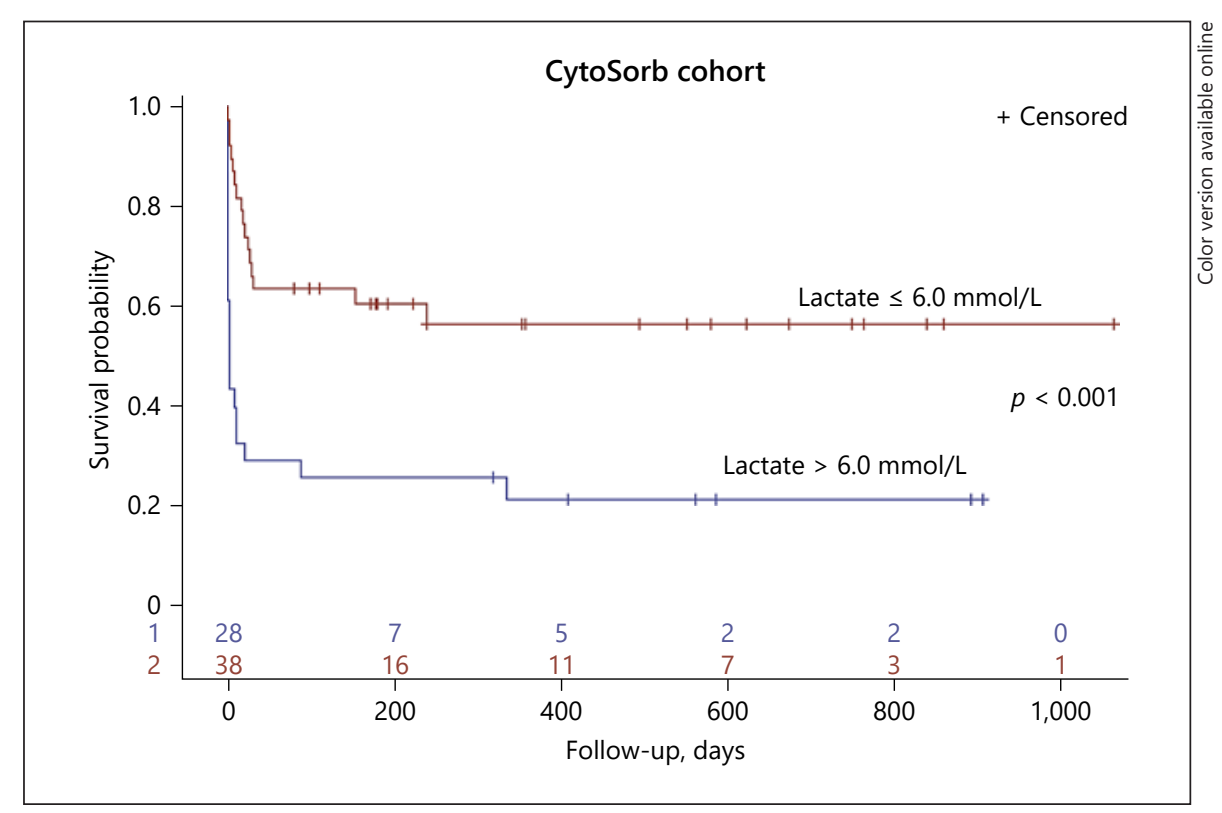

Table 2. Cox regression analysis for all-cause mortality in CytoSorb-treated patients

\begin{tabular}{|c|c|c|c|c|c|c|}
\hline Variables at start of therapy & $\operatorname{HR}(95 \% \mathrm{CI})$ & $p$ value & $\mathrm{HR}(95 \% \mathrm{CI})$ & $p$ value & HR (95\% CI) & $p$ value \\
\hline Age per 10 years older & $1.53(1.16-2.01)$ & 0.003 & - & & - & \\
\hline Any comorbidity* & $2.97(1.35-6.52)$ & 0.007 & - & & - & \\
\hline Yes, age per 10 years older & - & & $2.53(1.12-5.72)$ & 0.025 & $2.60(1.22-5.52)$ & 0.013 \\
\hline Female gender & $0.89(0.47-1.68)$ & 0.715 & - & & - & \\
\hline Body mass index & $0.97(0.92-1.02)$ & 0.213 & - & & - & \\
\hline CRRT duration & $0.94(0.86-1.03)$ & 0.201 & - & & - & \\
\hline Log creatinine & $0.76(0.25-2.25)$ & - & - & & - & \\
\hline SOFA & $1.15(1.03-1.29)$ & 0.014 & $1.08(0.95-1.22)$ & 0.275 & - & \\
\hline Admission ICU to treatment & $1.01(0.91-1.11)$ & 0.913 & - & & - & \\
\hline Admission hospital to treatment & $1.02(0.96-1.09)$ & 0.517 & - & & - & \\
\hline Surgery & $0.57(0.29-1.14)$ & 0.113 & $0.96(0.42-2.21)$ & 0.924 & - & \\
\hline \multicolumn{7}{|l|}{ Type of sepsis } \\
\hline Pneumosepsis & $1.69(0.81-3.51)$ & 0.159 & $0.78(0.29-2.12)$ & 0.623 & - & \\
\hline Abdominal sepsis & $0.62(0.32-1.19)$ & 0.152 & $0.41(0.18-0.98)$ & 0.044 & $0.34(0.17-0.70)$ & 0.004 \\
\hline
\end{tabular}

CRRT, continuous renal replacement therapy; MAP, mean arterial pressure; ICU, intensive care unit. Any comorbidity $(n=43,64 \%)$ : hypertension $(n=23,34 \%)$, prior heart failure $(n=4,6 \%)$, chronic kidney (any KDIGO class) disease including hemodialysis $(n=8$, $12 \%)$, peripheral artery disease $(n=10,15 \%)$, chronic obstructive lung disease $(n=8,12 \%)$, diabetes mellitus type $2(n=14,21 \%)$, coronary artery disease $(n=9,13 \%)$, and cerebrovascular accident $(n=6,9 \%)$. Note that these factors on their own do not show significance in these analyses. ${ }^{*} p$ value $=0.029$ for interaction between comorbidity and age. 
compared to CRRT alone. In CytoSorb-treated patients, it was observed that age, lactate level at the start of therapy, presence of any comorbidity, and abdominal sepsis were factors associated with long-term and also short-term allcause mortality. This is the first study to show that the benefit achieved with CytoSorb in the first month of ICU admission is enduring in the long term and that a lactate level above $6.0 \mathrm{mmol} / \mathrm{L}$ is an important determinant of outcome.

It is currently not known what the best timing for CytoSorb therapy in septic shock is. In the current study, we have shown that lower levels of lactate or noradrenaline were associated with an improved outcome. A lactate level above $6.0 \mathrm{mmol} / \mathrm{L}$ had a positive predictive value for long-term all-cause mortality of almost $80 \%$. Indeed, this is a retrospective analysis and confounding by indication may have an impact on the current data. Nevertheless, it was observed that patients more often were treated with CytoSorb as a last-resort option when lactate and noradrenaline levels were already (too) high, as $>50 \%$ of the CytoSorb-treated patients already had lactate levels above $6.0 \mathrm{mmol} / \mathrm{L}$ at the start of treatment. In line with our findings, a current report also showed that initiation of CytoSorb within $48 \mathrm{~h}$ of admission was associated with better outcome [16]. Given these lines of evidence, it seems reasonable to suggest that CytoSorb should be initiated earlier in the disease course rather than in case of refractory sepsis with high lactatemia. The timing should be further evaluated in future studies.

As was shown in our previous publication, before applying weights CytoSorb-treated patients were not comparable to those treated with CRRT alone at the start of therapy as they had worse hemodynamic characteristics including higher levels of lactate, need for vasopressors, lower MAP, and higher SOFA scores. Nonetheless, they still had a similar outcome to those treated with CRRT alone. Indeed patients treated with CRRT more often showed chronic kidney disease; however, this factor was not associated with long-term outcome in our analysis. When we applied IPTW adjusting for MAP, it was shown that CytoSorb-treated patients had an improved survival. Presence of chronic kidney disease, male gender, or other factors (peripheral artery disease or COPD) which were at imbalance did not show significant associations with outcome.

The current data show that, not unexpectedly, the first days of ICU admission were most important in the determination of clinical outcome. It is important to underline that the 28-day mortality rate showed to be a good surrogate for overall outcome as $>80 \%$ of patients who survived up to 28 days after ICU admission also survived in the long term. There are some important limitations of the current study. Next to the retrospective nature of the study, we did not have inflammatory cytokines such as IL-6 or procalcitonin levels available. In addition, although this is currently the largest cohort study of septic shock patients treated with CytoSorb analyzed for mortality, the number of patients assessed is still relatively small and the results are partly obtained from a propensity score analysis. Moreover, we were not able to perform an analysis for fluid balances as this would entail a dynamic analysis and this falls outside the scope of the current article. The results obtained from this study should be further confirmed in randomized clinical trials.

In conclusion, we have shown that the survival achieved with both CRRT and CytoSorb for patients with septic shock leads to an improved outcome beyond 28 days of ICU admission and may be enhanced for CytoSorb treatment. Importantly, lactate levels above $6.0 \mathrm{mmol} / \mathrm{L}$ were predictive for mortality with a $79 \%$ specificity and $80 \%$ positive predictive value. Therefore, for patients with septic shock not responding to fluid therapy and with a lactate level below $6.0 \mathrm{mmol} / \mathrm{L}$, CytoSorb therapy should be considered, especially for young patients with abdominal sepsis. Future randomized trials should take into account this lactate level since too high lactate levels are associated with poor treatment response.

\section{Statement of Ethics}

All patients, their relatives, or legal representatives gave their informed consent for the admission to the ICU and to undergo specific interventions. Patients were not recontacted for the purpose of this study. Recontacting patients for informed consent for the current retrospective chart study would introduce a serious bias because a large proportion is out of follow-up or deceased. All procedures were carried out per standard of care per protocol. This retrospective cohort study was performed in accordance with the protocol, the principles of the Declaration of Helsinki 1964 as modified by the 64th WMA General Assembly, Fortaleza, Brazil, October 2013, with notes of clarification on paragraphs 29 and 30 added by the WMA General Assembly, and the local national laws governing the conduct of clinical research studies. This study was approved by the ethics committee of the Maasstad Hospital Rotterdam, the Netherlands (MZ0582-07-14 and L2017-030).

\section{Conflict of Interest Statement}

W.P.B. and S.D. have received speaker's fee from CytoSorb. C.I. has received a grant from CytoSorb to commence a randomized controlled trial on the effect of the adsorber on the microcirculation of critically ill patients at the Department of Intensive Care of the Erasmus Medical Center, Rotterdam. 


\section{Funding Sources}

The authors did not receive any funding.

\section{Author Contributions}

W.P.B.: study design, data collection, statistical methods and analysis, writing of the manuscript, and approval of the final version. S.D. and C.I.: study design, critical review of the manuscript, and approval of the final version. W.P.B., S.D., and C.I. had full access to all of the data in the study and take responsibility for the integrity of the data and the accuracy of the data analysis.

\section{References}

1 Lozano R, Naghavi M, Foreman K, Lim S, Shibuya K, Aboyans V, et al. Global and regional mortality from 235 causes of death for 20 age groups in 1990 and 2010: a systematic analysis for the global burden of disease study 2010. Lancet. 2012 Dec 15;380(9859):2095128.

2 Vincent JL, Marshall JC, Namendys-Silva SA, François B, Martin-Loeches I, Lipman J, et al. Assessment of the worldwide burden of critical illness: the intensive care over nations (ICON) audit. Lancet Respir Med. 2014 May; 2(5):380-6.

3 Fleischmann C, Scherag A, Adhikari NK, Hartog CS, Tsaganos T, Schlattmann P, et al. Assessment of global incidence and mortality of hospital-treated sepsis. Current estimates and limitations. Am J Respir Crit Care Med. 2016 Feb 1;193(3):259-72.

4 Rudd KE, Johnson SC, Agesa KM, Shackelford KA, Tsoi D, Kievlan DR, et al. Global, regional, and national sepsis incidence and mortality, 1990-2017: analysis for the global burden of disease study. Lancet. 2020 Jan 18; 395(10219):200-11.

5 Levy MM, Artigas A, Phillips GS, Rhodes A, Beale R, Osborn T, et al. Outcomes of the surviving sepsis campaign in intensive care units in the USA and Europe: a prospective cohort study. Lancet Infect Dis. 2012 Dec;12(12): 919-24.

6 Singer M, Deutschman CS, Seymour CW, Shankar-Hari M, Annane D, Bauer M, et al. The third international consensus definitions for sepsis and septic shock (sepsis-3). JAMA. 2016 Feb 23;315(8):801-10.

7 Bellomo R, Tipping P, Boyce N. Continuous veno-venous hemofiltration with dialysis removes cytokines from the circulation of septic patients. Crit Care Med. 1993 Apr;21(4):5226.

8 Hoffmann JN, Faist E. Removal of mediators by continuous hemofiltration in septic patients. World J Surg. 2001 May;25(5):651-9.

9 Rimmele T, Kellum JA. Clinical review: blood purification for sepsis. Crit Care. 2011;15(1): 205.

10 Brouwer WP, Duran S, Kuijper M, Ince C. Hemoadsorption with CytoSorb shows a decreased observed versus expected 28-day allcause mortality in ICU patients with septic shock: a propensity-score-weighted retrospective study. Crit Care. 2019 Sep 18;23(1): 317.

11 Houschyar KS, Pyles MN, Rein S, Nietzschmann I, Duscher D, Maan ZN, et al. Continuous hemoadsorption with a cytokine adsorber during sepsis - a review of the litera- ture. Int J Artif Organs. 2017 May 29;40(5): 205-11.

12 Schadler D, Pausch C, Heise D, Meier-Hellmann A, Brederlau J, Weiler N, et al. The effect of a novel extracorporeal cytokine hemoadsorption device on IL-6 elimination in septic patients: a randomized controlled trial. PLoS One. 2017;12(10):e0187015.

13 Gruda MC, Ruggeberg KG, O'Sullivan P, Guliashvili T, Scheirer AR, Golobish TD, et al. Broad adsorption of sepsis-related PAMP and DAMP molecules, mycotoxins, and cytokines from whole blood using CytoSorb ${ }^{\circ}$ sorbent porous polymer beads. PLoS One. 2018;13(1): e0191676.

14 Friesecke S, Stecher SS, Gross S, Felix SB, Nierhaus A. Extracorporeal cytokine elimination as rescue therapy in refractory septic shock: a prospective single-center study. J Artif Organs. 2017 Sep;20(3):252-59.

15 Robins JM, Hernán MA, Brumback B. Marginal structural models and causal inference in epidemiology. Epidemiology. 2000 Sep; 11(5):550-60.

16 Mehta Y, Mehta C, Kumar A, George JV, Gupta A, Nanda S, et al. Experience with hemoadsorption CytoSorb ${ }^{\oplus}$ in the management of septic shock patients. World J Crit Care Med. 2020 Jan 31;9(1):1-12. 